\title{
NUMERICAL INVESTIGATION OF POOL NUCLEATE BOILING IN NANOFLUID WITH LATTICE BOLTZMANN METHOD
}

\author{
Afsaneh Rostamzadeh, Khosrow Jafarpur, Ebrahim Goshtsbi Rad \\ School of Mechanical Engineering, Shiraz University, Shiraz, Iran \\ e-mail:af_rostamzadeh@yahoo.com; kjafarme@shirazu.ac.ir; goshtasb@shirazu.ac.ir
}

\begin{abstract}
Due to significant improvement of thermal performance and other properties of nanofluids, this group of liquids is in high demand. According to the literature, the effect of nanoparticles on boiling heat transfer enhancement or degradation is not the same among different investigations. In the present article, the pseudo-potential multiphase lattice Boltzmann method is used to simulate nucleate pool boiling with two different fluids: a pure liquid and a nanofluid. The current results indicate that the contact angle is the same for both the fluid and nanofluid when the vapor bubble detachment occurs. Also, bubble departure diameter is greater in the base liquid while bubble release frequency is higher in the nanofluid. In brief, the present results demonstrate that using a nanofluid instead of its base fluid will increase the boiling heat transfer coefficient.
\end{abstract}

Keywords: pool boiling, nanofluid, lattice Boltzmann method, heat transfer

\section{Introduction}

Boiling and two phase flow phenomena are observed in many industrial processes and applications, such as refrigeration, air-conditioning, energy conversion systems, heat exchange systems, thermochemical processes, cooling of high-power electronic components, cooling of nuclear reactors, food, as well as space applications (Barber et al., 2011). Enhancement of boiling heat transfer may improve energy efficiency and achieves significant reduction of energy consumption. In other words, for industrial boiling systems, the enhancement of boiling heat transfer may cause an enormous increase in the power level of boilers without any increase in size or operating temperature. For this purpose, many researchers used nanofluids to enhance boiling heat transfer and convective boiling performance (Ahn et al., 2010; Cheng, 2009; Cheng et al., 2008; Das et al., 2003; Kim, 2007; Kwark et al., 2010; Lee and Mudawar, 2007; Taylor and Phelan, 2009; Wang and Mujumdar, 2007; You et al., 2003). Nanofluids are practical liquid suspensions containing particles that are smaller than $100 \mathrm{~nm}$. With some enhanced properties, they have wide potential applications for intensifying heat transfer and energy efficiency in a variety of systems (Huminic and Huminic, 2011; Kole and Dey, 2012; Park et al., 2009; Zeinali Heris, 2011). However, the study of nanofluid two-phase flow and thermal physics is still in the initial stages. Considering the lack of theoretical knowledge of the underlying mechanism of boiling heat transfer with and without nanoparticles, further investigations in this field of research are exceedingly in demand. Basically, this phenomenon is very complicated because of nonlinearity of the boiling process. Besides, in some numerical models such as Level Set and Volume of fluid (VOF) methods (Hirt and Nichols, 1981; Osher and Sethian, 1988), the interface tracking process increases the complexity of the problem and the computational costs.

As an effective numerical approach, the Lattice Boltzmann Method (LBM) has been successfully applied to simulate fluid flow and transport phenomena. Unlike conventional CFD methods, the LBM is based on microscopic models and mesoscopic kinetic equations. The main advantages of LBM are the simplicity of programming and the parallelism of the algorithm. Therefore, 
this method is an appropriate technique for modeling single component hydrodynamics, multiphase and multicomponent flows (Sukop and Thorne, 2007). Researchers have developed LBM to find a precise model for multiphase flows, such as the color function model (Rothman and Keller, 1988), the interaction potential model (Shan and Chen, 1993) and the free energy model (Swift et al., 1996). Their new models have been successful to some extent. To model multiphase problems, there are some defects in traditional CFD methods. For example, the difficulty in the implementation of interface tracking between immiscible phases, but the LBM is capable of incorporating these interactions without tracking. In addition to this important property, no assumptions or empirical correlations are used in the LBM method. Moreover, the lattice Boltzmann studies for nucleate boiling have been performed by some researchers as well. They have investigated the vapor bubble behavior and claimed that their numerical results could be considered as a basic work or reference for application of boiling performances (Cheng et al., 2010; Dong et al., 2010; Hazi and Markus, 2009; Inamuro et al., 2004; Jain and Tentner, 2009; Liu et al., 2010; Sun et al., 2013; Yang et al., 2001; Zhang and Chen, 2003; Zheng et al., 2006).

Although, a significant number of researches exist on the pool boiling of nanofluids focused on heat transfer performance including the critical heat flux and heat transfer coefficient, the investigations of the nanoparticle influence on the bubble dynamics are quite limited. Also these studies do not fully explain the mechanism responsible for the augmentation or deterioration of the heat transfer coefficient in nanofluids, and some of them are inconsistent as well. However, most of the previous works have described the surface modification or the interaction between the nanoparticles and liquid vapor interface (Phan et al., 2010; Vafaei and Wen, 2010). Vafaei and Wen (2010) examined dynamics of a gas bubble in a nanofluid made of gold nanoparticles. The bubble was not formed by boiling but gas was injected in the liquid with a nozzle. They demonstrated that the nanoparticles significantly affect the bubble growth. The bubble growth during boiling has been studied using a pure liquid on nano coated surfaces; this was the work done by Phan et al. (2010). Effect of surface wettability was investigated for several surfaces with various nano-coatings. It was found that a higher surface wettability increases the bubble departure radius and decreases the bubble release frequency. Therefore, in this study, the multiphase (liquid and vapor phases) lattice Boltzmann method based on a pseudo-potential model is applied to simulate pool-boiling nanofluids. Moreover, the current work examines dynamic growth of vapor bubbles in a nanofluid as well as the governing thermal effects. The effective parameters such as growth time, waiting time, bubble release frequency and bubble detachment diameter, which govern the heat transfer from the heater surfaces to the boiling fluids are obtained and compared for both pure liquids and nanofluids. Besides, the heat transfer performance for nanofluids relative to base fluids is studied as well.

\section{Model description}

\subsection{Dynamic and thermal Lattice Boltzmann model}

The density distribution function and the temperature distribution function are needed to simulate the phase change process in the LBM method. In the case that forces and potentials act on the particles, there are some methods to incorporate the force term in the LBM model (Shan and Chen, 1993; Guo et al., 2002; Buick and Greated, 2000). Kupershtokh (2004) showed that the Exact Difference Method (EDM) has better accuracy compared with other methods. Therefore, the evolution equation of the density distribution function is written as follows (Kupershtokh, 2004)

$$
f_{i}\left(\mathbf{x}+\mathbf{e}_{i} \Delta t, t+\Delta t\right)-f_{i}(\mathbf{x}, t)=-\frac{1}{\tau}\left[f_{i}(\mathbf{x}, t)-f_{i}^{e q}(\mathbf{x}, t)\right]+\Delta f_{i}(\mathbf{x}, t)
$$


where $f_{i}(\mathbf{x}, t)$ is the particle distribution function, $\mathbf{e}_{i}$ is the lattice velocity vector; $\tau$ is the velocity relaxation time, $\Delta t$ is the time step, $\Delta f_{i}(\mathbf{x}, t)$ is the force term. The equilibrium distribution function is taken as

$$
f_{i}^{e q}=\omega_{i} \rho\left[1+3 \frac{\mathbf{e}_{i} \mathbf{u}}{c^{2}}+\frac{9}{2} \frac{\left(\mathbf{e}_{i} \mathbf{u}\right)^{2}}{c^{4}}-\frac{3}{2} \frac{\mathbf{u}^{2}}{c^{2}}\right]
$$

In Eq. (2.2), $c=\Delta x / \Delta t$ is the lattice speed ( $\Delta x$ is the lattice spacing) and $\omega_{i}$ are the weight coefficients. For $\mathrm{D}_{2} \mathrm{Q}_{9}$ scheme, the weighting coefficients $\omega_{i}$ are given by $\omega_{0}=4 / 9, \omega_{1-4}=1 / 9$ and $\omega_{5-8}=1 / 36$. The discrete lattice velocity vector $\mathbf{e}_{i}$ is given as

$$
\mathbf{e}_{i}= \begin{cases}(0,0) & i=0 \\ ( \pm 1,0) c,(0, \pm 1) c & i=1, \ldots, 4 \\ ( \pm 1, \pm 1) c & i=5, \ldots, 8\end{cases}
$$

The force term in EDM is calculated from

$$
\Delta f_{i}(\mathbf{x}, t)=f_{i}^{e q}(\rho, \mathbf{u}+\Delta u)-f_{i}^{e q}(\rho, \mathbf{u})
$$

where $\Delta \mathbf{u}=(\Delta t / \rho) \mathbf{F}$ is the velocity change due to the force term. The density and velocity of the fluid are given by

$$
\rho(\mathbf{x}, t)=\sum_{i} f_{i}(\mathbf{x}, t) \quad \rho(\mathbf{x}, t) \mathbf{u}(\mathbf{x}, t)=\sum_{i} f_{i}(\mathbf{x}, t) \mathbf{e}_{i}
$$

The evolution equation of the temperature distribution function is

$$
g_{i}\left(\mathbf{x}+\mathbf{e}_{i} \Delta t, t+\Delta t\right)-g_{i}(\mathbf{x}, t)=-\frac{1}{\tau_{\theta}}\left[g_{i}(\mathbf{x}, t)-g_{i}^{e q}(\mathbf{x}, t)\right]+\omega_{i} \phi \Delta t
$$

In Eq. (2.6), $\tau_{0}$ is the relaxation time; $\phi$ is the source term which is responsible for the phase change derived by Gong and Cheng (2012). Then, the equation for the phase change is as follows

$$
\phi=T\left[1-\frac{1}{\rho c_{v}}\left(\frac{\partial p}{\partial T}\right)_{\rho}\right] \nabla \cdot \mathbf{U}
$$

$g_{i}^{e q}$ is the equilibrium distribution function for temperature given by

$$
g_{i}^{e q}=\omega_{i} T\left[1+3 \frac{\mathbf{e}_{i} \mathbf{U}}{c^{2}}+\frac{9}{2} \frac{\left(\mathbf{e}_{i} \mathbf{U}\right)^{2}}{c^{4}}-\frac{3}{2} \frac{\mathbf{U}^{2}}{c^{2}}\right]
$$

When any force exists in the system, the real fluid velocity is modified by $\rho \mathbf{U}=\sum_{i} \mathbf{e}_{i} f_{i}+\mathbf{F} \Delta t / 2$. Also, the temperature is obtained as follows

$$
T(\mathbf{x}, t)=\sum_{i} g_{i}(\mathbf{x}, t)
$$

Moreover, the thermal diffusivity and kinematic viscosity are calculated from

$$
\alpha=c^{2}\left(\tau_{\theta}-\frac{1}{2}\right) \frac{\Delta t}{3} \quad \nu=c^{2}\left(\tau-\frac{1}{2}\right) \frac{\Delta t}{3}
$$

In the current work, $\mathbf{F}$ represents multiphase flow and is a resultant vector of interparticle interaction forces. It contains forces responsible for phase separation, gravity and interaction between the solid surface and the fluid. 


\subsubsection{Multiphase lattice Boltzmann model}

In this investigation, the pseudo-potential proposed by Shan and Chen (1993) is used. The separation of different phases microscopically is due to interaction between the molecules of a fluid (Shan and Doolen, 1995). The interparticle interaction force can be expressed as (Yuan and Schaefer, 2006)

$$
\mathbf{F}_{\text {int }}(\mathbf{x}, t)=-c_{0} g \psi(\mathbf{x}, t) \nabla \psi(\mathbf{x}, t)
$$

where $c_{0}$ is a constant depending on the lattice structure, and for the $\mathrm{D}_{2} \mathrm{Q}_{9}$ model it is equal to 6.0. The coefficient for the strength of the interparticle force is $g$, with $g>0$ representing a repulsive force between the particles and $g<0$ an attractive force. $\psi(\mathbf{x})$ is called "effective mass" which is defined as a function of $\mathbf{x}$ through its dependency on the local density, and is specified by the equation of state with the following equation for present simulation

$$
\psi(\rho)=\sqrt{\frac{2(p-\rho / 3)}{c_{0} g}}
$$

The modified expression for the interparticle interaction force proposed by Gong and Cheng (2012) is used to improve the accuracy of the multiphase model

$$
\mathbf{F}_{\text {int }}(\mathbf{x})=-\beta c_{0} \psi(\mathbf{x}) g \nabla \psi(\mathbf{x})-\frac{1-\beta}{2} c_{0} g \nabla \psi^{2}(\mathbf{x})
$$

where $\beta$ is the weighting factor (for Peng-Robinson equation of state, $\beta=1.16$ ).

For numerical evaluation of the gradient term $\nabla \psi$ in a $\mathrm{D}_{2} \mathrm{Q}_{9}$ lattice, both nearest and nextnearest sites have been used, which gives a six-point scheme for two dimensions, i.e.

$$
\begin{aligned}
& \frac{\partial \psi(i, j)}{\partial x}=c_{1}[\psi(i+1, j)-\psi(i-1, j)] \\
& \quad+c_{2}[\psi(i+1, j+1)-\psi(i-1, j+1)+\psi(i+1, j-1)-\psi(i-1, j-1)] \\
& \frac{\partial \psi(i, j)}{\partial y}=c_{1}[\psi(i, j+1)-\psi(i, j-1)] \\
& \quad+c_{2}[\psi(i+1, j+1)-\psi(i+1, j-1)+\psi(i-1, j+1)-\psi(i-1, j-1)]
\end{aligned}
$$

$c_{1}$ and $c_{2}$ are weighting coefficients for the nearest and next nearest sites, respectively. In this article, $c_{1}=4 c_{2}=1 / 3$ proposed by Yuan and Schaefer (2006) have been used.

In addition to the interparticle forces, if the problem includes a solid wall boundary, it is essential to consider the forces between the fluid particles and surfaces. This interaction force can also model the wettability of the surface with the fluid, defined as

$$
F_{a d s}(\mathbf{x})=-G_{a d s} \psi(\mathbf{x}) \sum_{i} \omega_{i} s\left(\mathbf{x}+\mathbf{e}_{i} \Delta t\right) \mathbf{e}_{i}
$$

The adsorption coefficient parameter $G_{a d s}$ (in Eq. (2.15)) controls the strength of the force between the wall and fluid; $s$ is the switch that takes a value of one if the site at $\mathbf{x}+\mathbf{e}_{i} \Delta t$ is a solid and is zero otherwise.

On the other hand, the gravity force is given by

$$
F_{\text {gravity }}(\mathbf{x})=\mathbf{G}\left[\rho(\mathbf{x})-\rho_{\text {ave }}\right]
$$

where $\mathbf{G}$ is the acceleration of gravity and $\rho_{\text {ave }}$ the average density of the whole computation domain at each time step. 


\subsubsection{Equation of state (EOS)}

According to the definition of the equation of state (EOS), if there is no interaction force, the fluid will behave like an ideal gas, however, by selecting a more realistic EOS, a better performance will be obtained from the LBE simulation. Yuan and Schaefer (2006) discussed a non-ideal EOS. Referring to those results, the Peng-Robinson EOS is more accurate for real gases. So, in this study, P-R EOS has been chosen; the constants of this equation have been set as $a=2 / 49, b=2 / 21$ and $R=1$

$$
p=\frac{\rho R T}{1-b \rho}-\frac{a \rho^{2} \varepsilon(T)}{1+2 b \rho-b^{2} \rho^{2}} \quad a=0.45724 \frac{R^{2} T_{c}^{2}}{P_{c}} \quad b=0.0779 \frac{R T_{c}}{P_{c}}
$$

where

$$
\varepsilon(T)=\left[1+\left(0.37464+1.54226 \omega-0.26992 \omega^{2}\right)\left(1-\sqrt{\frac{T}{T_{c}}}\right)\right]^{2}
$$

with $\omega$ as the acentric factor.

At the critical point, $\partial P_{c} / \partial \rho_{c}=0$ and $\partial^{2} P_{c} / \partial \rho_{c}^{2}=0$ should be satisfied (and therefore $T_{c}=0.0729$ and $\left.P_{c}=0.0595\right)$. In the current investigation, dimensionless variables such as $T / T_{c}, \rho / \rho_{c}$ and all other variables are chosen based on the lattice unit. As it was previously presented (Liu and Cheng, 2013), the liquid-vapor interface is defined as the location where the density is at $\rho_{i}=\left(\rho_{l}+\rho_{v}\right) / 2$.

\subsection{Nanofluid properties}

The properties of nanofluids may be defined in terms of $\varphi$ (volume fraction of nanoparticles), thermo-physical properties of nanoparticles and pure liquid.

The effective dynamic viscosity of the nanofluid $\mu_{n f}$ in the present work has been calculated based on the model given by Brinkman (Brinkman, 1952)

$$
\mu_{n f}=\frac{\mu_{f}}{(1-\varphi)^{2.5}}
$$

where $\mu_{f}, \varphi$ are viscosity of fluid and volume fraction of the particles, respectively.

Also, the effective density of the nanofluid is obtained from

$$
\rho_{n f}=(1-\varphi) \rho_{f}+\varphi \rho_{p}
$$

In Eq. (2.19), $\rho_{n f}, \rho_{f}$ and $\rho_{p}$ are the density of the nanofluids, base fluid and nanoparticles, respectively.

For the specific heat capacity of the nanofluid $C p_{n f}$ at constant pressure, the following expression is used

$$
\rho_{n f} C p_{n f}=(1-\varphi) \rho_{f} C p_{f}+\varphi \rho_{p} C p_{p}
$$

$C p_{f}$ and $C p_{p}$ in Eq. (2.20, are the specific heat capacities at constant pressure for the base fluid and nanoparticles, respectively.

The thermal conductivity of nanofluid knf is determined by (Hamilton and Crosser, 1962)

$$
\frac{k_{n f}}{k_{f}}=\frac{k_{p}+2 k_{f}-2 \varphi\left(k_{f}-k_{p}\right)}{k_{p}+2 k_{f}+\varphi\left(k_{f}-k_{p}\right)}
$$

where $k_{f}$ is thermal conductivity of water and $k_{p}$ is thermal conductivity of nanoparticles. 


\section{Results and discussion}

\subsection{Coexistence curve and surface tension}

First, simulation has been done for a single component multiphase flow to assess validity of the proposed model. A $150 \times 150$ lattice structure and periodic boundary condition were chosen for all directions. A bubble (droplet) with radius of 5 lattice nodes was placed in the center. Simulation proceeded until equilibrium was reached. Densities of the vapor and liquid are shown in Fig. 1. Analytical solution given by the Maxwell construction is also displayed. It is clear that the liquid and vapor branches of the coexistence curve are reproduced quite well. $T_{r}$ and $\rho_{r}$ represent the reduced temperature and reduced density, respectively, which were defined the same as the dimensionless temperature and density.

In addition, if the initial density is set to be randomly distributed around the critical density, phase separation will occur for temperatures below the critical value. After 40000 time steps, the steady state is reached and the phase separation ultimately leads to a single droplet in the vapor phase or vice versa.

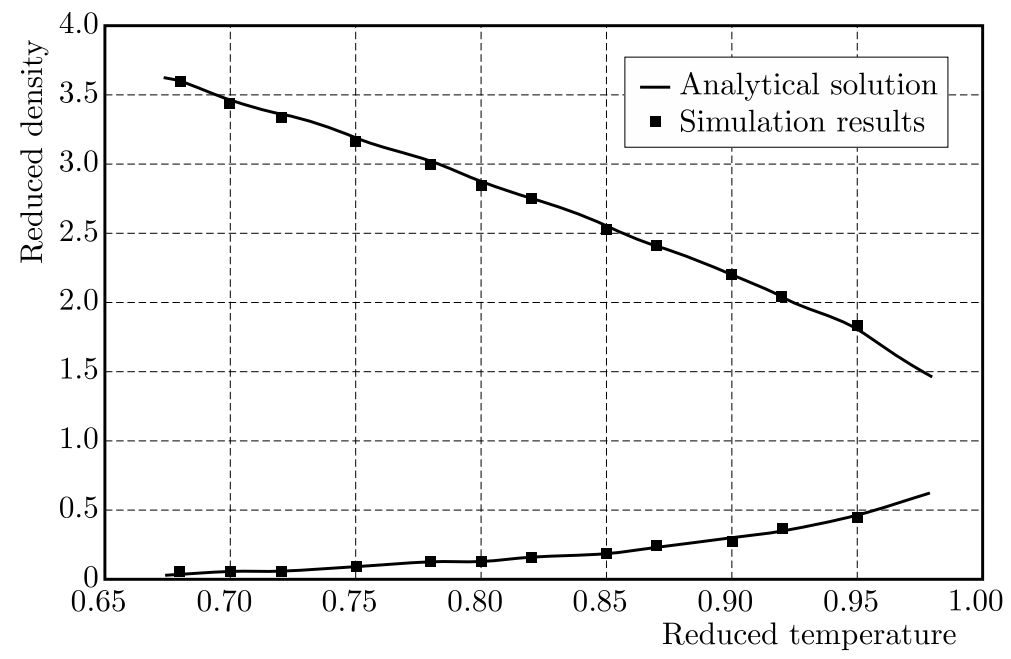

Fig. 1. Coexistence curve

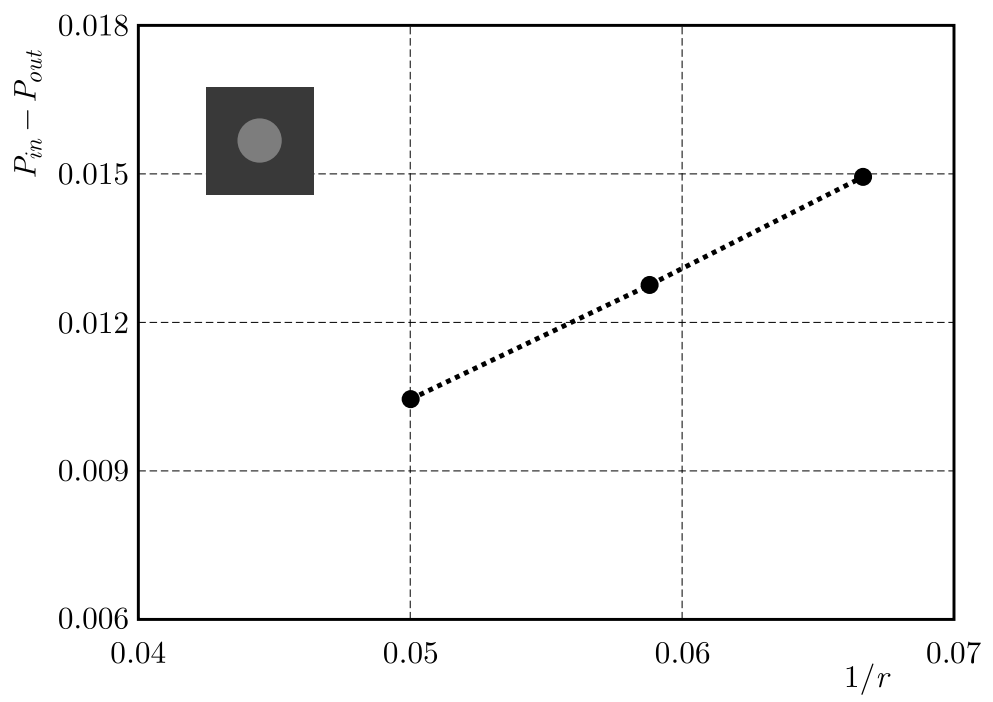

Fig. 2. Comparison of the simulation results with the Laplace equation

Also, the surface tension coefficient for the P-R equation of state at different temperatures is calculated by measuring the density and finding the pressure difference inside and outside the 
bubble (droplet) using the EOS. The results shown in Fig. 2 indicate a linear dependence of the inverse radius on the pressure difference for the liquid. According to the Laplace law, for the $2 \mathrm{D}$ bubble (droplet) one can estimate the surface tension given as follows: $\Delta p=\sigma / R$.

\subsection{Bubble growth and departure in pool boiling for pure liquid}

To simulate bubble growth and its departure from the heated horizontal thermal surface in pool boiling, a rectangular domain with periodic lateral directions is used. A non-slip solid wall with constant temperature is employed at the bottom of the domain. Constant temperature and constant pressure (corresponding to the saturated pressure of $T_{b u l k}$ ) are specified at the top boundary. A $150 \times 450$ lattice structure is generated for computation purposes. By applying different mesh sizes, the bubble departure and bubble growth are independent of the mesh size. Initially, the computational domain has been occupied by the liquid phase at the temperature $T_{\text {bulk }}=0.82 T_{c}$, and the temperature $T_{w}$ has been specified as $0.88 T_{c}$ at the wall boundary.

A spherical bubble with a radius of three lattice units has been initially located at coordinates $(75,0)$. The present numerical model requires some setting in the parameters before the start of bubble growth, e.g. dimensionless gravity to be set as $G=+0.00002$ in the $y$ direction. Also, by varying the parameter $G_{a d s}$, which specifies the strength of the contribution force between the fluid particle and surfaces, the complete range of contact angles can be obtained. For example, $G_{a d s}=-0.1$ corresponds to $90^{\circ}$ contact angle. The nondimensional time step has been created by length and velocity scales as follows

$$
l_{0}=\sqrt{\frac{\sigma}{G\left(\rho_{L}-\rho_{V}\right)}} \quad \mathbf{U}_{0}=\sqrt{G l_{0}} \quad t^{*}=\frac{t U_{0}}{l_{0}}
$$

where $\sigma$ is the surface tension.

Profiles of vapor bubble growth and departure from a heated wall are shown in Fig. 3. The growth and departure of the vapor bubble from the solid heating surface are dynamic processes for which the momentum and energy exchanges between the growing bubble and the surrounding liquid must be considered. At the first stage, the growth of the bubble requires a certain amount of energy from the heating surface to vaporize the surrounding liquid. In the next stage, the bubble departure is determined by the net forces acting on the bubble during its growth. Buoyancy has often played a major role between the forces that act on the bubble such as surface tension and dynamic forces.
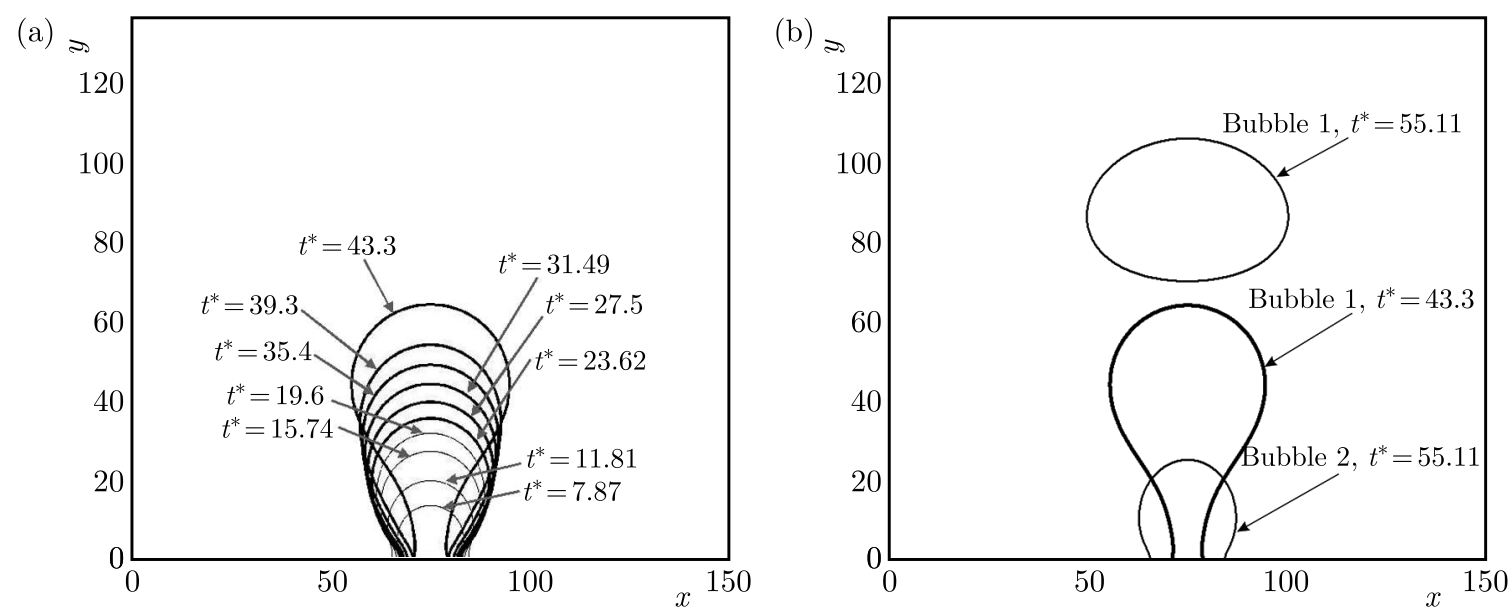

Fig. 3. Bubble (a) expanding and (b) departure process 
In Fig. 4, vapor bubble streamlines and interfaces are shown before and after its detachment. The dynamic behavior of the bubble is growth, departure and rise due to the action of buoyancy. These are clearly shown in Figs. 3 and 4. It is noted that the induced velocity causes transformation of the natural convection process into localized forced convection and increases heat transfer by moving the cold fluid towards the superheated wall. It is found that the simulation results of the bubble dynamics and growth pattern shown in Figs. 3 and 4 are similar to the results of the available experimental data of Mukherjee and Dhir (2004).
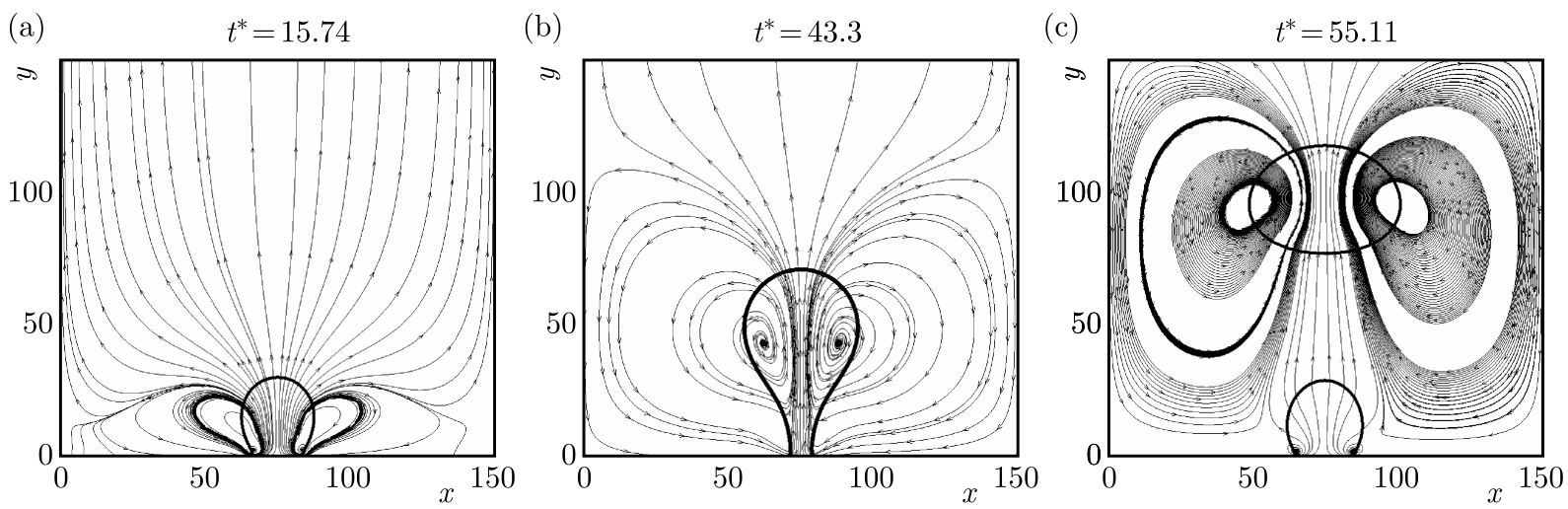

Fig. 4. Flow fields at various time (a) $t^{*}=15.74$, (b) $t^{*}=43.3$, (c) $t^{*}=55.11$

In the early stage, the growth of the vapor bubble is influenced by the over-pressure force and controlled by the inertia as shown in Fig. 4a at $t^{*}=15.74$. Following this, the growth proceeds until the buoyancy force overcomes the adhesion forces, which is shown in Fig. 4b and finally the bubble departs (Fig. 4c).

Figure 5 indicates the relationship between the detachment diameters and the gravity acceleration. The equivalent diameter for the growing bubble has been calculated according to a hypothetical sphere with the same volume as the original bubble shape. The bubble departure diameter is proportional to the inverse of the square root of the gravity acceleration. These calculated results are in good agreement with other correlations previously presented by Fritz (1935) and Phan et al. (2010). Their analytical analyses showed that the bubble departure diameter is proportional to $G^{-0.5}$ while the current LBM outcomes predict the variation of bubble departure diameter to be proportional to $G^{-0.51}$.

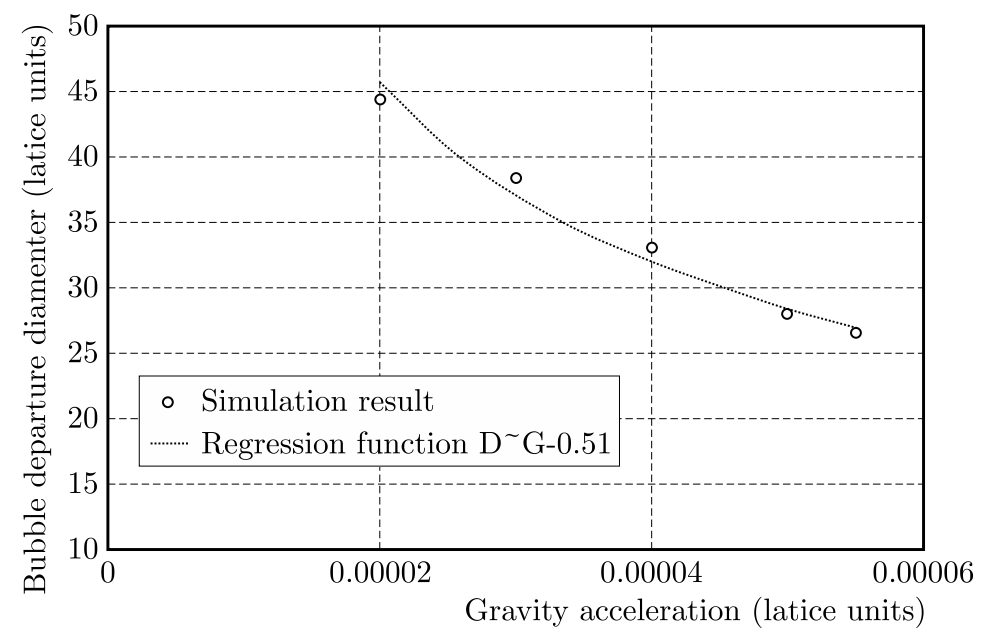

Fig. 5. Change in the bubble departure vs. gravity acceleration 


\subsection{Dynamic growth of a bubble in a nanofluid}

In the investigation of two-phase flow and boiling phenomena of nanofluids, aluminum oxide nanoparticles are chosen because of their well-recorded thermal properties and widespread use in such types of research. In this study, the nanofluid is assumed as one-component. On the assumption of no slip and thermal equilibrium between the base fluid and nanoparticles, the properties of nanofluids can be defined in terms of the ratio of the solid volume fraction of the particles in a pure fluid $\varphi$. So, thermo-physical properties of the nanofluid such as thermal conductivity, viscosity, liquid density and specific heat capacity are calculated based on equations described in Section 2.2. Moreover, it is assumed that the nanoparticles do not have an important role in the interparticle forces, consequently, the EOS for nanofluid can be considered equal to that of the pure liquid (Kim et al., 2006; Kim et al., 2007).

In the present work, the base fluid is water and $1 \%$ volume fraction of nanoparticles is added to the base fluid in order to investigate the effect of the bubble growth dynamics and heat transfer. Thermo-physical properties of the selected nanoparticles and water at $20^{\circ} \mathrm{C}$ are reported in Table 1.

Table 1. Thermo-physical properties of water and nanoparticles

\begin{tabular}{|l|c|c|c|c|}
\hline & $\rho\left[\mathrm{kg} / \mathrm{m}^{3}\right]$ & $C_{p}[\mathrm{~J} /(\mathrm{kg} \mathrm{K})]$ & $k[\mathrm{~W} /(\mathrm{m} \mathrm{K})]$ & $\alpha \cdot 10^{7}\left[\mathrm{~m}^{2} / \mathrm{s}\right]$ \\
\hline \hline Water (Bejan, 2013) & 997.1 & 4179 & 0.613 & 1.47 \\
\hline $\mathrm{Al}_{2} \mathrm{O}_{3}$ (Abu-Nada, 2009) & 3890 & 775 & 31.8 & 105 \\
\hline
\end{tabular}

\subsection{Bubble release frequency}

When a bubble starts to grow on a heating surface, a specific amount of time (growing time $t_{g}$ ) is required until the bubble departs from the surface. On the other hand, a time interval, called the waiting period $t_{w}$ is the duration between the release of one bubble to the nucleation of the next at a given nucleation site. Accordingly, the bubble frequency is defined as $f=1 /\left(t_{g}+t_{w}\right)$.

The present numerical results show that growing time and waiting period for nanofluids are shorter than in their base pure liquids. For example, the times for bubble dynamics in nanofluids are $t^{*}=11.81102,27.55906$, and 43.66929, respectively, and the flow patterns are similar to the bubble dynamics in the liquid phase as shown in Fig. 4. It is obvious that these time values are much lower than in pure liquids, which leads to an increase in the bubble release frequency of the nanofluid. Reduction of these times for the nanofluids relative to the base liquids for different time steps at the growing process is 25,36 , and $21 \%$, respectively. Also, at this condition the bubble release frequency for the nanofluid is $27 \%$ higher than for the base liquid.

Figure 6 depicts a comparison between numerical results of the time variation of bubble diameter for the pure liquid and nanofluid with $1 \%$ volume fraction of aluminum oxide nanoparticles. It appears that using the nanofluid makes the process of bubble growth and departure faster than in the base fluid. As the time passes, the variation in diameter between the nanofluid and the base fluid increases. Also the detachment diameter for the nanofluid is smaller than in the base liquid, which is consistent with the increase in the bubble release frequency. This phenomenon has been observed for all gravity accelerations investigated in this work.

The growth time of a vapor bubble for the nanofluid and the pure liquid is shown in Fig. 7 . Differences in the growth time between the nanofluid and the base fluid decrease with the increasing acceleration of gravity. In fact, buoyancy is the force that overcomes surface tension at the bubble base and pulls the bubble from the surface. Moreover, the change of bubble departure with gravity acceleration for the nanofluid is similar to that of the base fluid as shown in Fig.5. However, it should be noted that the bubble radius at the time of detachment is decreased for the nanofluid in comparison to the pure fluid for all gravity accelerations. Reduction of the 


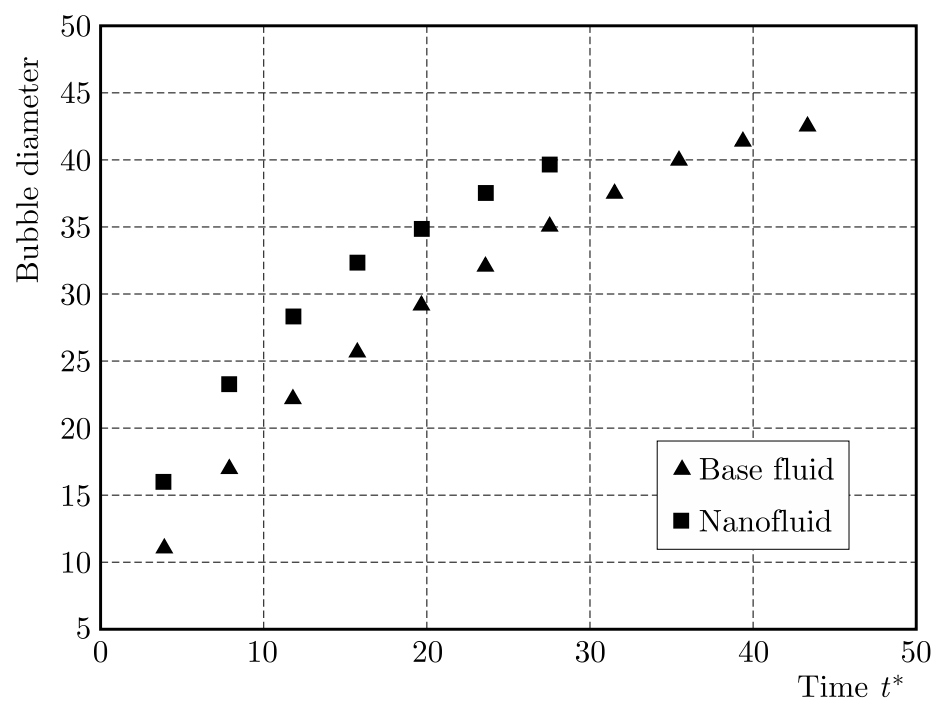

Fig. 6. Bubble diameter variation for the nanofluid and base liquid

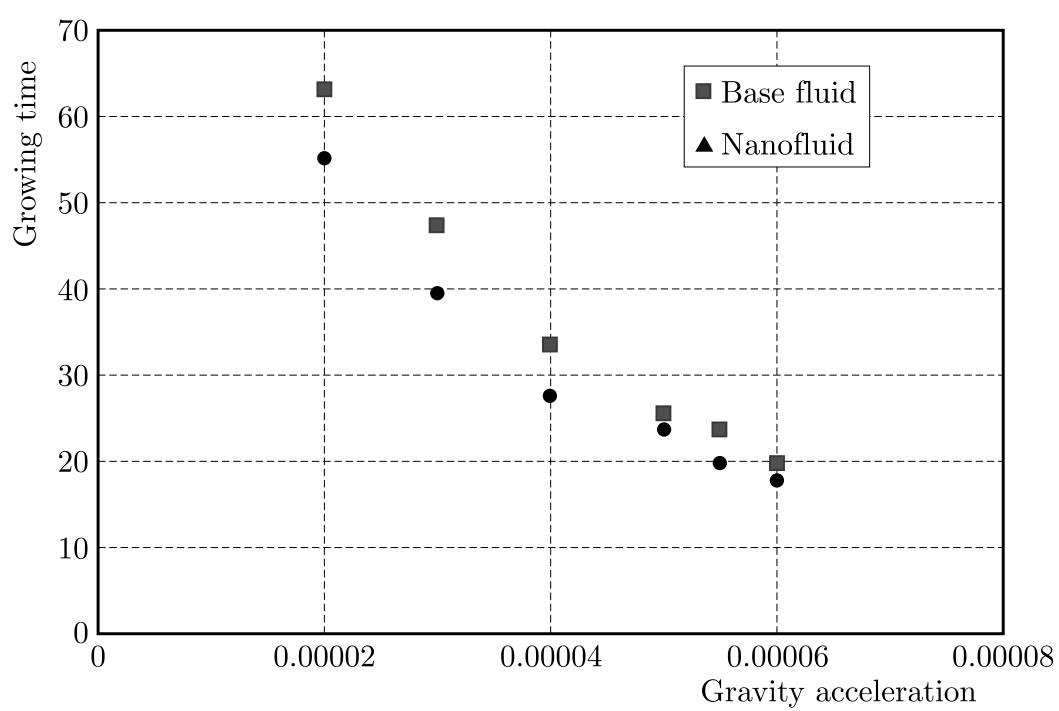

Fig. 7. Plot of growing time versus gravity acceleration for the nanofluid and base fluid (water)

departure diameter for the bubble with the gravity acceleration for the nanofluid is slightly higher than that for the base liquid.

In the current investigation, problems that possibly occur in experimental works such as particle deposition do not exist. In other words, on this assumption, the bubble dynamics is independent of the heating surface or at least this factor does not have an effective role. Indeed, the deposition of particles on the heating surface could change surface roughness. This could lead to an increase in the number of nucleation sites or, conversely, it could reduce thermal conductivity that provides a barrier to heat transfer and decreases bubble generation. Thus, this explanation can be one of the reasons for inconsistency in the previous researches. The contact angles of the detaching vapor bubble for the nanofluid and base fluid with the same $G_{a d s}$ and acceleration of gravity, are similar (see Fig. 3).

It is possible to estimate the power absorbed by a bubble during its growth. The power required to grow a bubble by evaporation for both the nanofluid and the base fluid is obtained from the following equation

$$
p(t)=\rho_{v} h_{l v} \frac{d V}{d t}=m^{\circ} h_{l v}
$$


According to thermodynamic relations, the specific latent heat $\left(h_{l v}\right)$ is derived by Gong and Cheng (2013) for any equation of state. The vaporization power calculated for nanofluids and water is shown in Fig. 8. From this figure, it is obvious that the power is increasing as the bubble grows for both the base fluid and nanofluid. At the beginning, the growing power for the nanofluid is greater than that of the base fluid. However, after a while, the bubble in the base fluid increases in volume, therefore the evaporation heat flux will be increased and, finally. the power heat fluxes for both the base fluid as well as the nanofluid become approximately equal. On the other hand, because the bubble release frequency for the nanofluid is greater than of the base fluid, eventually the nanofluid will absorb more power than the base fluid.

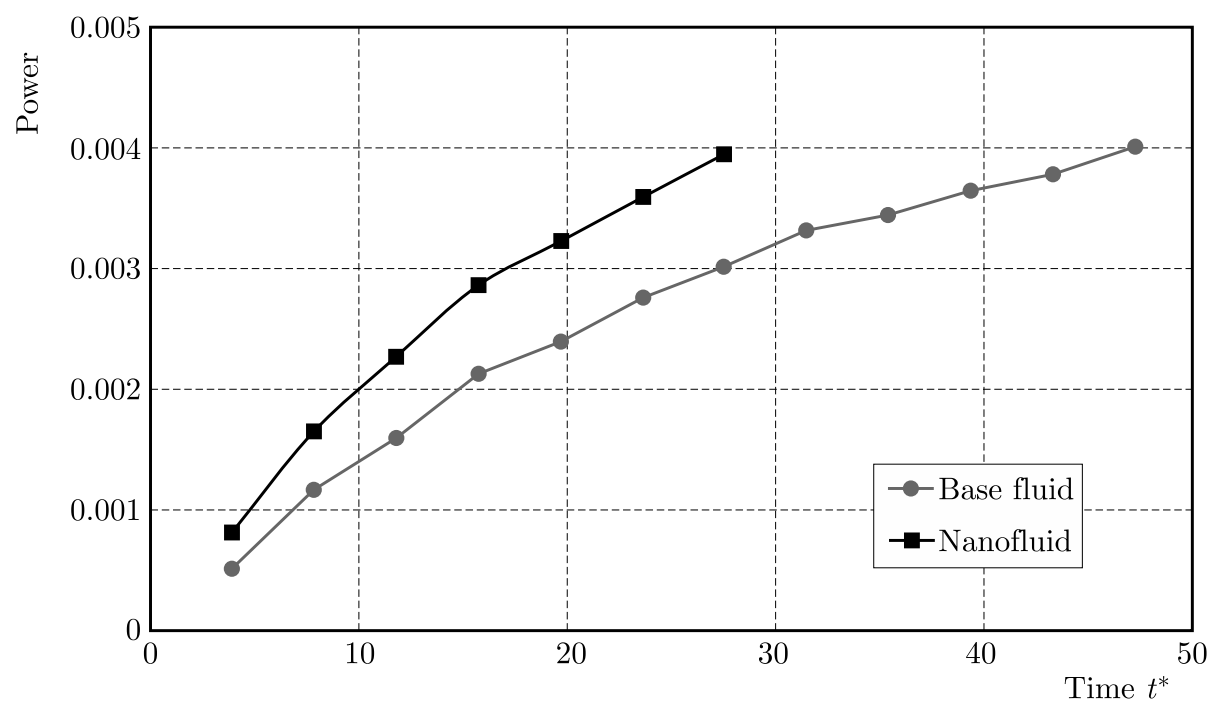

Fig. 8. Power required for vaporization

In the present work, the heat transfer coefficients during nucleate pool boiling have been investigated as well. The heat transfer coefficient associated with the boiling process is computed from the following equation

$$
h_{\text {avg }}=\frac{1}{A} \int_{A} \frac{q}{\Delta T} d A \quad q=\left.k_{\text {eff }} \frac{\partial T}{\partial y}\right|_{w}
$$

where $k_{\text {eff }}$ is thermal conductivity of the nanofluid. The heat transfer coefficient could be averaged over the area and time. Time averaging is calculated during one cycle, from the initial stage to bubble detachment.

The heat transfer coefficient enhancement compared to the base fluid is displayed in Fig. 9. By increasing the temperature difference, the increment in the heat transfer coefficient tends to be larger, although a slight increase in the heat transfer coefficient with temperature difference has been observed. The maximum enhancement of the heat transfer coefficient is $18 \%$. The main reason for the increase of the heat transfer coefficient is the modification of thermo-physical properties of the nanofluid. For example, these properties such as thermal conductivity and thermal diffusivity of the nanofluid are improved and enhanced in comparison to the base fluid. From these results, the heat transfer in microlayer vaporization is greater and leads to an increase in the growth of the vapor bubble. In this work, the nanofluid is considered homogeneous, therefore, in the microlayer aggregation of nanoparticles does not occur relative to other regions, and thermo-physical properties in all regions are identically developed. Furthermore, in the previous experimental researches, it has been shown that the suspended nanoparticles in the base liquid decrease the radius of the bubble and, therefore, more active sites on the heating 
surface occur. Consequently, the boiling heat transfer coefficient increases, which is consistent with the present results (Raveshi et al., 2013; Kim and Kim, 2009; Yang and Liu, 2011).

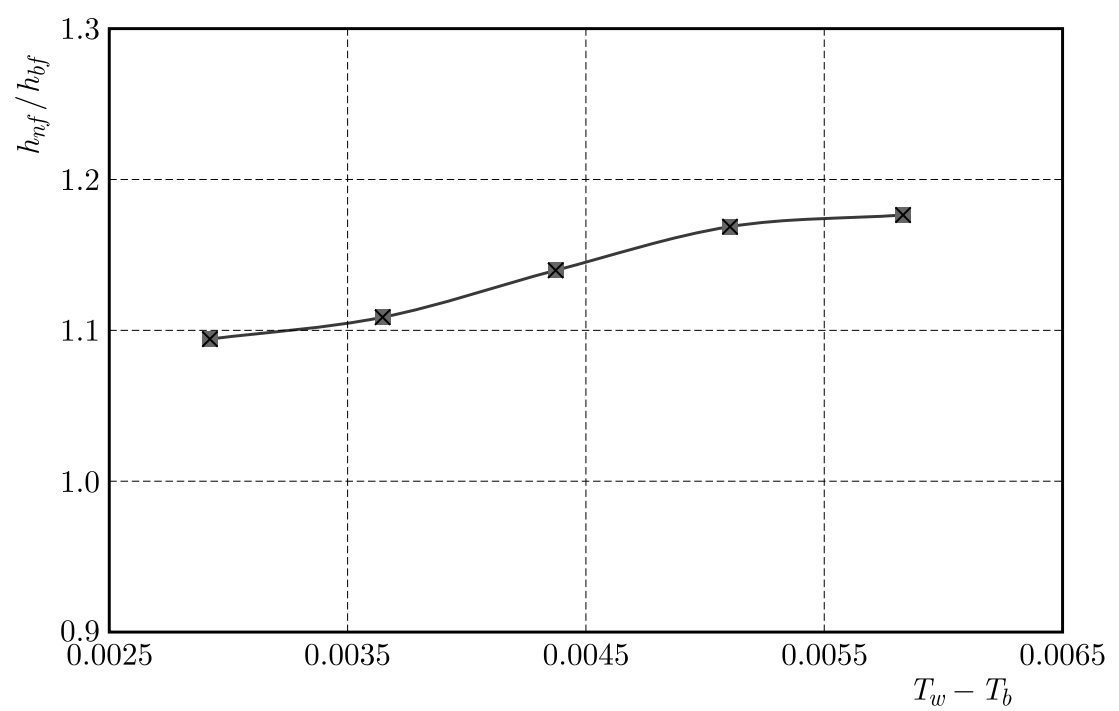

Fig. 9. Enhancement of the heat transfer coefficient versus $\Delta T$

\section{Conclusion}

In this research, based on the pseudo-potential multiphase lattice Boltzmann method as well as the modified LBM thermal model, the bubble growth and departure from a horizontal heated surface are investigated for a pure liquid and a nanofluid. The comparison of the present results with those of analytical studies reveals that the current numerical results are in good agreement with the analytical solution.

To answer the question of whether nanofluids can cause the enhancement of pool boiling heat transfer performance or not bubble growth, the diameter of departure, contact angle and bubble release frequency of nanofluid are examined in the present work. By comparing the results of nucleate pool boiling for a $1 \%$ volume fraction nanofluid and those for the base fluid, the following results are observed. The bubble diameter at detachment is smaller for the nanofluid in comparison to its base liquid. Also, the power heat flux absorbed by the vapor bubble in the nanofluid is greater than in the pure liquid. Moreover, the bubble release frequency is higher for the nanofluid. In addition, the contact angle has been found to be similar for both fluids. Considering the above results, it is concluded that all three phenomena, smaller growing and waiting time, improved thermo-physical properties and smaller bubble departure diameter in the nanofluid, enhance the heat transfer coefficient.

\section{References}

1. Abu-Nada E., 2009, Effects of variable viscosity and thermal conductivity of $\mathrm{Al}_{2} \mathrm{O}_{3}$-water nanofluid on heat transfer enhancement in natural convection, International Journal of Heat and Fluid Flow, 30, 4, 679-90

2. Ahn H.S. , Kim H., Jo H., Kang S., Chang W., Kim M.H., 2010, Experimental study of critical heat flux enhancement during forced convective flow boiling of nanofluid on a short heated surface, International Journal of Multiphase Flow, 36, 5, 375-84

3. Barber J., Brutin D., TAdrist L., 2011, A review on boiling heat transfer enhancement with nanofluids, Nanoscale Research Letters, 6, 1, 1-16 
4. Bejan A., 2013, Convection Heat Transfer, John Wiley \& Sons

5. Brinkman H., 1952, The viscosity of concentrated suspensions and solutions, The Journal of Chemical Physics, 20, 4, 571

6. Buick J., Greated C., 2000, Gravity in a lattice Boltzmann model, Physical Review E, 61, 5, 5307

7. Cheng L., 2009, Nanofluid heat transfer technologies, Recent Patents on Engineering, 3, 1, 1-7

8. Cheng L., Bandarra F., Enio P., Thome J.R., 2008, Nanofluid two-phase flow and thermal physics: a new research frontier of nanotechnology and its challenges, Journal of Nanoscience and Nanotechnology, 8, 7, 3315-3332

9. Cheng M., Hua J., Lou J., 2010, Simulation of bubble-bubble interaction using a lattice Boltzmann method, Computers and Fluids, 39, 2, 260-70

10. Das S. K., Putra N., Roetzel W., 2003, Pool boiling characteristics of nano-fluids, International Journal of Heat and Mass Transfer, 46, 5, 851-862

11. Dong Z., Li W., Song Y., 2010, A numerical investigation of bubble growth on and departure from a superheated wall by lattice Boltzmann method, International Journal of Heat and Mass Transfer, 53, 21, 4908-4916

12. Fritz W., 1935, Maximum volume of vapor bubbles, Physik Zeitschr, 36, 11, 379-84

13. Gong S., Cheng P., 2012, A lattice Boltzmann method for simulation of liquid-vapor phase-change heat transfer, International Journal of Heat and Mass Transfer, 55, 17, 4923-7

14. Gong S., Cheng P., 2013, Lattice Boltzmann simulation of periodic bubble nucleation, growth and departure from a heated surface in pool boiling, International Journal of Heat and Mass Transfer, 64, 122-132

15. Guo Z., Zheng C., Shi B., 2002, Discrete lattice effects on the forcing term in the lattice Boltzmann method, Physical Review E, 65, 4, 046308

16. Hamilton R., Crosser O., 1962, Thermal conductivity of heterogeneous two-component systems, Industrial and Engineering Chemistry Fundamentals, 1, 3, 187-191

17. Hazi G., Markus A., 2009, On the bubble departure diameter and release frequency based on numerical simulation results, International Journal of Heat and Mass Transfer, 52, 5, 1472-1480

18. Hirt C.W., Nichols B.D., 1981, Volume of fluid (VOF) method for the dynamics of free boundaries, Journal of Computational Physics, 39, 1, 201-225

19. Huminic G., Huminic A., 2011, Heat transfer characteristics of a two-phase closed thermosyphons using nanofluids, Experimental Thermal and Fluid Science, 35, 3, 550-557

20. Inamuro T., Ogata T., Ogino F., 2004, Numerical simulation of bubble flows by the lattice Boltzmann method, Future Generation Computer Systems, 20, 6, 959-964

21. Jain P.K., Tentner A., 2009, A lattice Boltzmann framework to simulate boiling water reactor core hydrodynamics, Computers and Mathematics with Applications, 58, 5, 975-986

22. Kim H., Kim M., 2009, Experimental study of the characteristics and mechanism of pool boiling CHF enhancement using nanofluids, Heat and Mass Transfer, 45, 7, 991-998

23. Kim S., Bang I. C., Buongiorno J., Hu L., 2006, Effects of nanoparticle deposition on surface wettability influencing boiling heat transfer in nanofluids, Applied Physics Letters, 89, 15, 153107

24. Kim S., Bang I.C., Buongiorno J., Hu L., 2007, Surface wettability change during pool boiling of nanofluids and its effect on critical heat flux, International Journal of Heat and Mass Transfer, 50, 19, 4105-4116

25. Kim S.J., 2007, Pool Boiling Heat Transfer Characteristics of Nanofluids, Massachusetts Institute of Technology 
26. Kole M., Dey T., 2012, Investigations on the pool boiling heat transfer and critical heat flux of ZnO-ethylene glycol nanofluids, Applied Thermal Engineering, 37, 112-119

27. Kupershtokn A., 2004, New method of incorporating a body force term into the lattice Boltzmann equation, Proceedings of 5th International EHD Workshop, University of Poitiers, Poitiers, France

28. Kwark S.M., Kumar R., Moreno G., Yoo J., You S.M., 2010, Pool boiling characteristics of low concentration nanofluids, International Journal of Heat and Mass Transfer, 53, 5, 972-981

29. Lee J., Mudawar I., 2007, Assessment of the effectiveness of nanofluids for single-phase and two-phase heat transfer in micro-channels, International Journal of Heat and Mass Transfer, 50, $3,452-463$

30. Liu X., Cheng P., 2013, Lattice Boltzmann simulation of steady laminar film condensation on a vertical hydrophilic subcooled flat plate, International Journal of Heat and Mass Transfer, 62, $507-514$

31. Liu M., Yu Z., Wang T., Wang J., FAn L.-S., 2010, A modified pseudopotential for a lattice Boltzmann simulation of bubbly flow, Chemical Engineering Science, 65, 20, 5615-5623

32. Mukherjee A., Dhir V., 2004, Study of lateral merger of vapor bubbles during nucleate pool boiling, Journal of Heat Transfer, 126, 6, 1023-1039

33. Osher S., Sethian J.A., 1988, Fronts propagating with curvature-dependent speed: algorithms based on Hamilton-Jacobi formulations, Journal of Computational Physics, 79, 1, 12-49

34. PARK K.-J., Jung D., Shim S.E., 2009, Nucleate boiling heat transfer in aqueous solutions with carbon nanotubes up to critical heat fluxes, International Journal of Multiphase Flow, 35, 6, $525-532$

35. Phan H.T., Caney N., Marty P., Colasson S., Gavillet J., 2010, Surface coating with nanofluids: the effects on pool boiling heat transfer, Nanoscale and Microscale Thermophysical Engineering, 14, 4, 229-244

36. Phan H.T., Caney N., Marty P., Colasson S., Gavillet J., 2010, A model to predict the effect of contact angle on the bubble departure diameter during heterogeneous boiling, International Communications in Heat and Mass Transfer, 37, 8, 964-969

37. Raveshi M.R., Keshavarz A., Mojarrad M.S., Amiri S., 2013, Experimental investigation of pool boiling heat transfer enhancement of alumina-water-ethylene glycol nanofluids, Experimental Thermal and Fluid Science, 44, 805-814

38. Rothman D.H., Keller J.M., 1988, Immiscible cellular-automaton fluids, Journal of Statistical Physics, 52, 3/4, 1119-1127

39. Shan X., Chen H., 1993, Lattice Boltzmann model for simulating flows with multiple phases and components, Physical Review E, 47, 3, 1815

40. Shan X., Doolen G., 1995, Multicomponent lattice-Boltzmann model with interparticle interaction, Journal of Statistical Physics, 81, 1/2, 379-393

41. Sukop M.C., Thorne D.T., 2007, Lattice Boltzmann Modeling: an Introduction for Geoscientists and Engineers, Springer

42. Sun T., Li W., YANG S., 2013, Numerical simulation of bubble growth and departure during flow boiling period by lattice Boltzmann method, International Journal of Heat and Fluid Flow, 44, 120-129

43. Swift M.R., Orlandini E., Osborn W., Yeomans J., 1996, Lattice Boltzmann simulations of liquid-gas and binary fluid systems, Physical Review E, 54, 5, 5041

44. Taylor R.A., Phelan P.E., 2009, Pool boiling of nanofluids: comprehensive review of existing data and limited new data, International Journal of Heat and Mass Transfer, 52, 23, 5339-5347

45. VAFAEI S., Wen D., 2010, Effect of gold nanoparticles on the dynamics of gas bubbles, Langmuir, 26, 10, 6902-6907 
46. Wang X.-Q., Mujumdar A.S., 2007, Heat transfer characteristics of nanofluids: a review, International Journal of Thermal Sciences, 46, 1, 1-19

47. Yang X.F., Liu Z.H., 2011, Pool boiling heat transfer of functionalized nanofluid under subatmospheric pressures, International Journal of Thermal Sciences, 50, 12, 2402-2412

48. Yang Z., Dinh T.-N., Nourgaliev R., Sehgal B., 2001, Numerical investigation of bubble growth and detachment by the lattice-Boltzmann method, International Journal of Heat and Mass Transfer, 44, 1, 195-206

49. You S., Kim J., KIM K., 2003, Effect of nanoparticles on critical heat flux of water in pool boiling heat transfer, Applied Physics Letters, 83, 16, 3374-3376

50. Yuan P., Schaefer L., 2006, Equations of state in a lattice Boltzmann model, Physics of Fluids, 18, 4, 042101

51. Zeinali Heris S., 2011, Experimental investigation of pool boiling characteristics of lowconcentrated $\mathrm{CuO}$ /ethylene glycol-water nanofluids, International Communications in Heat and Mass Transfer, 38, 10, 1470-1473

52. Zhang R., Chen H., 2003, Lattice Boltzmann method for simulations of liquid-vapor thermal flows, Physical Review E, 67, 6, 066711

53. Zheng H., Shu C., Chew Y.-T., 2006, A lattice Boltzmann model for multiphase flows with large density ratio, Journal of Computational Physics, 218, 1, 353-371

Manuscript received March 25, 2015; accepted for print November 27, 2015 